\title{
FIBRE TENSOR PRODUCT BUNDLES
}

\author{
BERNARD R. GELBAUM AND ATHANASIOS KYRIAZIS
}

\begin{abstract}
In analogy with fibre bundles, which are locally Cartesian products, fibre tensor product bundles are objects that are locally tensor products. These can be patched together via transition maps, etc., into an object very similar to the set of sections of a locally convex algebra bundle.
\end{abstract}

0. Introduction. It is known $[4,5]$ that for large classes of topological algebras the following kind of relationships obtain: $\operatorname{Hom}\left(A \otimes_{C} B, C\right)$ is the pull-back of the diagram:

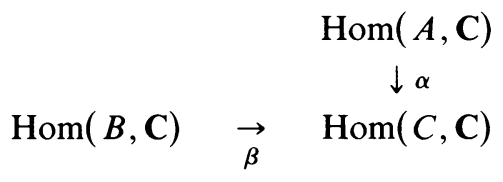

When $C=\mathbf{C}$, the pull-back is $\operatorname{simply} \operatorname{Hom}(A, \mathbf{C}) \times \operatorname{Hom}(B, \mathbf{C})$. Furthermore $[\mathbf{5}, \mathbf{7}$, $10]$ if $\mathscr{E}$ is a Banach algebra bundle (base space $X$ compact Hausdorff, projection $p$ : $\mathscr{E} \rightarrow X$, fibre $A$ a commutative or $Q$-uniform Banach algebra, open cover $\left\{U_{\lambda}\right\}$ of $X$, transition maps $\left.h_{\lambda \mu}: U_{\lambda} \cap U_{\mu} \rightarrow \operatorname{Aut}(A)\right)$, then the set $\Gamma$ of sections $\gamma: X \rightarrow \mathscr{E}$ is a Banach algebra and $\operatorname{Hom}(\Gamma, \mathbf{C})$ is also a fibre bundle (base space $X$, projection $\pi$ : $\operatorname{Hom}(\Gamma, \mathbf{C}) \rightarrow X$, fibre $\operatorname{Hom}(A, \mathbf{C})$, open cover $\left\{U_{\lambda}\right\}$ of $X$, transition maps $H_{\lambda \mu}$ : $\left.U_{\lambda} \cap U_{\mu} \rightarrow \operatorname{Auteo}(\operatorname{Hom}(A, \mathbf{C}))\right)$. For each $U_{\lambda},\left.\Gamma\right|_{U_{\lambda}}$ is a subalgebra of $\mathscr{C}\left(U_{\lambda}, A\right)$ which, in turn, is closely related to some kind of tensor product $\mathscr{C}\left(U_{\lambda}\right) \otimes A$. Thus, one is led to regard $\Gamma$ as having a "fibre bundle-like" structure, that is locally like a tensor product. In turn, one is led thus to the notion of a fibre tensor product bundle and the study of such an object in the context of topological algebra.

1. Fibre tensor product bundles. Let $A$ and $B$ be two unital locally convex algebras such that $\operatorname{Hom}(A, \mathbf{C})=$ spectrum of $A$ is compact. Associated with $A$ and $B$ are the following:

(i) In $B$, a finite set $\left\{I_{\lambda}\right\}$ of closed 2-sided ideals, having compact hulls $\left\{h\left(I_{\lambda}\right)=\right.$ $\left.F_{\lambda}\right\} \subset \operatorname{Hom}(B, \mathbf{C})$, the interiors $\left\{F_{\lambda}{ }^{\circ}=U_{\lambda}\right\}$ are nonempty and $\bigcup_{\lambda} U_{\lambda}=\operatorname{Hom}(B, \mathbf{C})$.

(ii) Continuous transition maps $\bar{h}_{\lambda \mu}: U_{\lambda} \cap U_{\mu} \rightarrow \operatorname{Aut}(A)=$ the group of continuous C-automorphisms of $A$; on $U_{\lambda} \cap U_{\mu} \cap U_{\nu}, \bar{h}_{\lambda \mu} \circ \bar{h}_{\mu \nu}=\bar{h}_{\lambda \nu}$; each $\bar{h}_{\lambda \mu}$ has a continuous extension $h_{\lambda \mu}: F_{\lambda} \cap F_{\mu} \rightarrow \operatorname{Aut}(A)$ behaving on $\left\{F_{\lambda}\right\}$ as the $\bar{h}_{\lambda \mu}$ behave on $\left\{U_{\lambda}\right\}$.

Received by the editors December 23, 1983.

1980 Mathematics Subject Classification. Primary 46M05.

(1985 American Mathematical Society $0002-9939 / 85 \$ 1.00+\$ .25$ per page 
(iii) The locally convex algebra direct sum $S$, namely if $B_{\lambda}=B / I_{\lambda}, S=\sum_{\lambda} B_{\lambda} \otimes_{\pi} A$, $\otimes_{\pi}$ denoting the projective tensor product $[\mathbf{8}]$; in $S$ a closed subalgebra $D$ consisting of all $\tau=\left(\tau_{\lambda}\right)$ in $S$ and such that if $F_{\lambda} \cap F_{\mu} \neq \varnothing$, then for some $f$ in $F_{\lambda} \cap F_{\mu}$ and $\tau_{\lambda}=\sum_{n} b_{\lambda n} \otimes a_{\lambda n}$,

$$
\sum_{n} \hat{b}_{\lambda n}(f) a_{\lambda n}=\sum_{n} \hat{b}_{\mu n}(f) h_{\lambda \mu} a_{\mu n} .
$$

The situations (i)-(iii) describe a coordinate fibre tensor product bundle $\mathscr{B}$. The object $D$, being the counterpart of the set of sections of an algebra bundle, is "locally" like $B_{\lambda} \otimes_{\pi} A$, and its localities are matched via the automorphisms $h_{\lambda \mu}$ in the manner described above.

The situation described above is exemplified if $A$ and $B$ are unital locally convex $Q$-algebras (locally convex algebras for which the group of units (invertible elements) is an open set [8]).

It will be shown that $\operatorname{Hom}(D, C)$ is (equivalent to) a kind of fibre bundle (base space $\operatorname{Hom}(B, \mathbf{C})$, projection $p: \operatorname{Hom}(D, \mathbf{C}) \rightarrow \operatorname{Hom}(B, \mathbf{C})$, fibre $\operatorname{Hom}(A, \mathbf{C})$, open cover $\left\{U_{\lambda}\right\}$, transition maps $h_{\mu \lambda}^{*}: U_{\lambda} \cap U_{\mu} \rightarrow \operatorname{Auteo}(\operatorname{Hom}(A, \mathbf{C}))=$ the group of self-homeomorphisms of $\operatorname{Hom}(A, \mathbf{C})$ ), equipped with a suitable topology, making it a toplogical transformation group and $h_{\mu \lambda}^{*}$ continuous (cf. comments after Lemma 1 in $\S 2$ below). Thus, the fibre tensor product is akin to the abstract algebraic version of the set $\Gamma$ of continuous sections of a Banach (or, more generally, locally convex [7]) algebra bundle.

\section{The theorems and their proofs.}

LeMMA 1. If $T$ is a C-automorphism of a locally convex algebra $A$, then $T^{*}$ : $\operatorname{Hom}(A, \mathbf{C}) \ni \chi \mapsto T^{*}(\chi) \in \operatorname{Hom}(A, \mathbf{C})\left(T^{*}(\chi) a=\chi(T(a))\right)$ is an auteomorphism of $\operatorname{Hom}(A, \mathbf{C})$.

Proof. The bijectivity of $T^{*}$ follows from that of $T$ and the bicontinuity of $T^{*}$ follows from the definition of the weak topology on $\operatorname{Hom}(A, \mathbf{C})$.

Denote by $\mathscr{A}^{*}$ the relevant part of $\mathscr{A} \equiv \operatorname{Auteo}(\operatorname{Hom}(A, \mathrm{C}))$ defined by the above lemma, i.e. $\mathscr{A}^{*}=\left\{T^{*}: T \in \operatorname{Aut}(A)\right\}$. Then since $\operatorname{Aut}(A)$ and $\mathscr{A}^{*}$ are bijectively related via $T \mapsto T^{*}, \mathscr{A}^{*}$ may be given the topology it inherits $\operatorname{from} \operatorname{Aut}(A)$ by this bijection, and then the continuity of $h_{\lambda \mu}: F_{\lambda} \cap F_{\mu} \rightarrow \operatorname{Aut}(A)$ implies the continuity of the (dual) maps $h_{\mu \lambda}^{*}: F_{\lambda} \cap F_{\mu} \rightarrow \mathscr{A}^{*}$. Now, it is assumed that $\operatorname{Aut}(A)$ is an equicontinuous subset of $\mathscr{L}_{s}(A)$ (the set of linear endomorphisms of $A ; \mathscr{L}_{s}(A)$ is topologized by convergence on finite sets). This assumption is valid, e.g., in case of a Fréchet locally convex algebra, when by $\operatorname{Aut}(A)$ one should mean, of course, auteomorphisms of the whole structure, namely isometries [2, Example 2, p. 22]. Hence $\operatorname{Aut}(A)$ is a topological transformation group. Furthermore, if $\mathscr{A}$ is equipped with the topology of convergence on compact sets, then $\mathscr{A}$ is a topological transformation group and the (dual) maps $h_{\mu \lambda}^{*}: F_{\lambda} \cap F_{\mu} \rightarrow \mathscr{A}$ are also continuous. Indeed, by $[10, \S 5.4$, p. 19] $\mathscr{A}$ is a topological transformation group and, moreover, $\mathscr{A}^{*}$ is also a topological transformation group since equicontinuity implies that the topologies of convergence on finite and on compact sets are the same $[2, \S 2.4$, Theorem 1, p. 29]. Thus, the transition maps $h_{\mu \lambda}^{*}: F_{\lambda} \cap F_{\mu} \rightarrow \operatorname{Auteo}(\operatorname{Hom}(A, \mathbf{C}))$ are, in fact, continuous. 
LeMMA 2. If $I_{\lambda}, I_{\mu}$ are closed ideals in the unital locally convex algebra $B$, then there is an injection

$$
\operatorname{Hom}\left(B / I_{\lambda} \otimes_{\pi} B / I_{\mu}, \mathrm{C}\right) \hookrightarrow \operatorname{hull}\left(I_{\lambda}+I_{\mu}\right)
$$

Proof. Since $B$ is unital (with identity $1_{B}$ ) the map $I_{\lambda} \ni x_{\lambda} \mapsto x_{\lambda} \otimes 1_{B}$ is an injection $I_{\lambda} \hookrightarrow I_{\lambda} \otimes B$ and in these circumstances $\left(I_{\lambda}+I_{\mu}\right.$ denoting the closed subspace spanned by $I_{\lambda}$ and $I_{\mu}$ ) there is an injection $I_{\lambda}+I_{\mu} \hookrightarrow I_{\lambda} \otimes B+B \otimes I_{\mu}$, whence there is a continuous epimorphism

$$
\psi: B / I_{\lambda}+I_{\mu} \rightarrow B / I_{\lambda} \otimes_{\pi} B+B \otimes_{\pi} I_{\mu} .
$$

On the other hand, since $B$ is unital there are bicontinuous isomorphisms

$$
B / I_{\lambda} \otimes_{\pi} B+B \otimes_{\pi} I_{\mu} \underset{\omega_{2}}{\rightarrow} B \otimes_{\pi} B / I_{\lambda} \otimes_{\pi} B+B \otimes_{\pi} I_{\mu} \underset{\omega_{1}}{\rightarrow} B / I_{\lambda} \otimes_{\pi} B / I_{\mu},
$$

as the following lines show.

In the following diagram $\pi_{2}, \pi_{2}^{\prime}, \pi_{\lambda}, \pi_{\mu}$ are the canonical quotient maps and $\varphi, \varphi^{\prime}$ are the canonical maps of the corresponding tensor products:

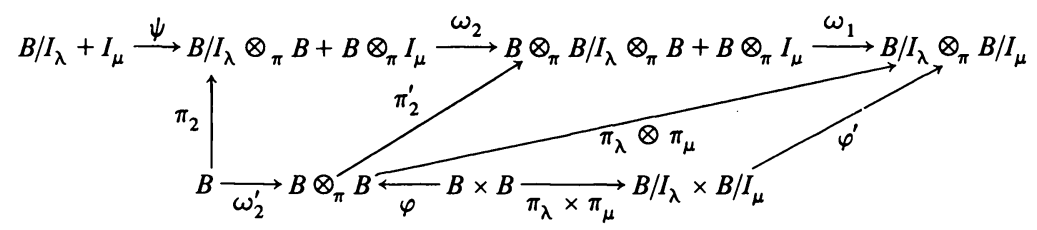

\section{Diagram 1}

Then $\omega_{1}$ is continuous iff $\omega_{1} \circ \pi_{2}^{\prime}$ is continuous and since $\omega_{1} \circ \pi_{2}^{\prime}=\pi_{\lambda} \otimes \pi_{\mu}, \omega_{1}$ is continuous; $\omega_{1}^{-1}$ is continuous iff $\omega_{1}^{-1} \circ \varphi^{\prime}$ is continuous iff $\left(\omega_{1}^{-1} \circ \varphi^{\prime}\right) \circ\left(\pi_{\lambda} \times \pi_{\mu}\right)$ is continuous; but $\left(\omega_{1}^{-1} \circ \varphi^{\prime}\right) \circ\left(\pi_{\lambda} \times \pi_{\mu}\right)=\left(\pi_{\lambda} \otimes \pi_{\mu}\right) \circ \varphi$ and so $\omega_{1}$ is bicontinuous. The bicontinuity of $\omega_{2}$ follows even more easily.

Finally,

$$
\operatorname{Hom}\left(B / I_{\lambda} \otimes_{\pi} B / I_{\mu}, \mathrm{C}\right) \underset{\text { (continuous) }}{\stackrel{\psi}{\rightarrow}} \operatorname{Hom}\left(B / I_{\lambda} \otimes_{\pi} B+B \otimes_{\pi} I_{\mu}, \mathrm{C}\right)
$$

and the latter is homeomorphic to $\operatorname{Hom}\left(B / I_{\lambda}+I_{\mu}, \mathbf{C}\right)=\operatorname{hull}\left(I_{\lambda}+I_{\mu}\right)$ [9].

THEOREM 1. If $\mathscr{B}$ is a fibre tensor product bundle, then $\operatorname{Hom}(D, \mathbf{C})$ may be identified with a kind of fibre bundle having base space $\operatorname{Hom}(B, \mathbf{C})$, fibre $\operatorname{Hom}(A, \mathbf{C})$ and group Auteo(Hom $(A, \mathbf{C}))$.

Proof. Since [8] $\operatorname{Hom}\left(B_{\lambda} \otimes_{\pi} A, \mathbf{C}\right)$ may be identified with $\operatorname{Hom}\left(B_{\lambda}, \mathbf{C}\right) \times$ $\operatorname{Hom}(A, \mathbf{C})$ and since $\operatorname{Hom}\left(B_{\lambda}, \mathbf{C}\right)=\operatorname{hull}\left(I_{\lambda}\right)=F_{\lambda}$ [9] there may be defined an equivalence relation $\sim_{\mathscr{B}}$ on the disjoint union $T=\dot{U}_{\lambda} F_{\lambda} \times \operatorname{Hom}(A, \mathbf{C})$ (*denoting dual):

$$
\left(f_{\lambda}, a_{\lambda}\right) \sim_{\mathscr{B}}\left(f_{\mu}, a_{\mu}\right) \text { iff }\left\{\begin{array}{c}
f_{\lambda}=f_{\mu} \in F_{\lambda} \cap F_{\mu} \\
\text { and } \\
a_{\lambda}=h_{\mu \lambda}^{*}\left(f_{\mu}\right)\left(a_{\mu}\right) .
\end{array}\right.
$$

If $p_{\lambda}^{*}: F_{\lambda} \times \operatorname{Hom}(A, \mathbf{C}) \rightarrow \operatorname{Hom}(D, \mathbf{C})$ is the dual of $p_{\lambda}: D \rightarrow B_{\lambda} \otimes_{\pi} A$, there may be defined a map $\nu: T \rightarrow \operatorname{Hom}(D, \mathbf{C})$ so that $\left.\nu\right|_{F_{\lambda} \times \operatorname{Hom}(A, C)}=p_{\lambda}^{*}$. It is directly verifiable 
that $\nu$ is continuous and compatible with $\sim_{\mathscr{B}}$, i.e., if $t_{1}, t_{2} \in T$ and $t_{1} \sim_{\mathscr{B}} t_{2}$, then $\nu\left(t_{1}\right)=\nu\left(t_{2}\right)$, whence $\nu$ defines a map

Thus, if $\pi_{\lambda}$ is the composition

$$
\vartheta: L \equiv T / \sim_{\mathscr{B}} \rightarrow \operatorname{Hom}(D, \mathbf{C}) .
$$

$$
F_{\lambda} \times \operatorname{Hom}(A, \mathbf{C}) \stackrel{i_{\lambda}}{\rightarrow} T \stackrel{\pi}{\rightarrow} L \equiv T / \sim_{\mathscr{B}},
$$

then $\vartheta \circ \pi_{\lambda}=p_{\lambda}^{*}$. The continuity of all maps considered with respect to the standard topologies is immediate. Furthermore, $\vartheta$ is injective as the following argument shows. [Note. Since $f_{\lambda} \in F_{\lambda}=\operatorname{hull}\left(I_{\lambda}\right), f_{\lambda}$ may be identified with an element, again denoted $f_{\lambda}$, of $\operatorname{Hom}\left(B_{\lambda}, \mathbf{C}\right)$. Conversely, an element $f_{\lambda}$ in $\operatorname{Hom}\left(B_{\lambda}, \mathbf{C}\right)$ may be identified with an element of $F_{\lambda}$ (in $\left.\operatorname{Hom}(B, \mathbf{C})\right)$.]

If $X=\pi_{\lambda}\left(f_{\lambda}, a_{\lambda}\right) \in L, Y=\pi_{\mu}\left(f_{\mu}^{\prime}, a_{\mu}^{\prime}\right) \in L$ and $\vartheta(X)=\vartheta(Y)$, then for $\tau=\left\{\tau_{\lambda}\right\}$ in $D,\left(f_{\lambda} \otimes a_{\lambda}\right)\left(\tau_{\lambda}\right)=\left(f_{\mu}^{\prime} \otimes a_{\mu}^{\prime}\right)\left(\tau_{\mu}\right)$. To show $X=Y$ it suffices to show $\left(f_{\lambda}, a_{\lambda}\right) \sim_{\mathscr{D}}$ $\left(f_{\mu}^{\prime}, a_{\mu}^{\prime}\right)$. However, $f_{\lambda}=f_{\mu}^{\prime}$ and their common value is in $\operatorname{hull}\left(I_{\lambda}\right) \cap \operatorname{hull}\left(I_{\mu}\right)=$ hull $\left(I_{\lambda}+I_{\mu}\right)$ iff for all $x$ in $B / I_{\lambda}+I_{\mu}, \hat{x}\left(f_{\lambda}\right)=\hat{x}\left(f_{\mu}^{\prime}\right)$. But if $g=\left(f_{\lambda} \otimes f_{\mu}^{\prime}\right)$ $\circ \omega_{1} \circ \omega_{2} \circ \omega_{2} \circ \psi$, then Diagram 1 may be completed as follows:

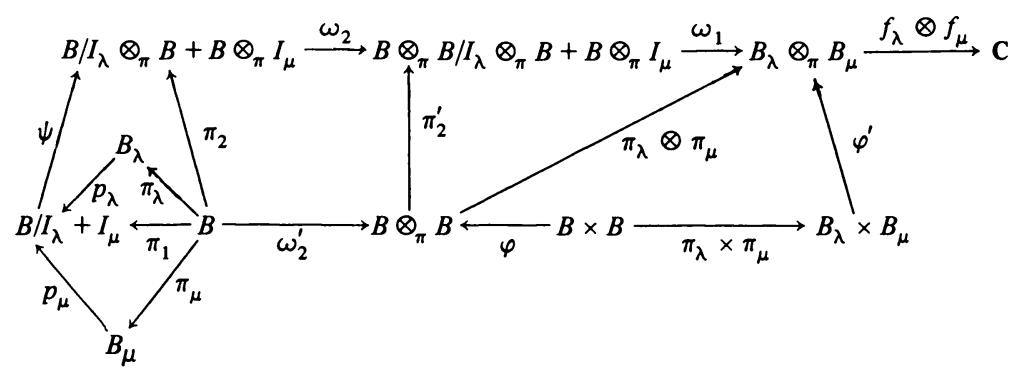

Diagram 2

. nus, $f_{\lambda}=g \circ p_{\lambda}$ and $f_{\mu}^{\prime}=g \circ p_{\mu}$ since if $z_{\lambda} \in B / I_{\lambda}$, then

$$
\begin{aligned}
f_{\lambda}\left(z_{\lambda}\right) & =\left(f_{\lambda} \otimes f_{\mu}^{\prime}\right)\left(z_{\lambda} \otimes 1_{B_{\mu}}\right)=\left(f_{\lambda} \otimes f_{\mu}^{\prime}\right)\left(\left(\pi_{\lambda} \otimes \pi_{\mu}\right)\left(z \otimes 1_{B}\right) \quad \text { (for some } z \text { in } B\right) \\
& =\left[\left(f_{\lambda} \otimes f_{\mu}^{\prime}\right) \circ\left(\pi_{\lambda} \otimes \pi_{\mu}\right) \circ \omega_{2}^{\prime}\right](z)=\left[\left(f_{\lambda} \otimes f_{\mu}^{\prime}\right) \circ \omega_{1} \circ \omega_{2} \circ \pi_{2}\right](z) \\
& =\left(g \circ p_{\lambda}\right)\left(\pi_{\lambda}(z)\right)=\left(g \circ p_{\lambda}\right)\left(z_{\lambda}\right),
\end{aligned}
$$

i.e., $f_{\lambda}=g \circ p_{\lambda}$ and similarly $f_{\mu}^{\prime}=g \circ p_{\mu}$. Since, for $x$ in $B / I_{\lambda}+I_{\mu}, x=p_{\lambda}\left(x_{\lambda}\right)=$ $p_{\mu}\left(x_{\mu}\right)$ it follows that $f_{\lambda}\left(x_{\lambda}\right)=f_{\mu}^{\prime}\left(x_{\mu}\right)$, i.e., $\hat{x}\left(f_{\lambda}\right)=\hat{x}\left(f_{\mu}^{\prime}\right), f_{\lambda}=f_{\mu}^{\prime} \equiv f \in \operatorname{hull}\left(I_{\lambda}\right) \cap$ $\operatorname{hull}\left(I_{\mu}\right)$.

By definition $f \neq 0$. If $a_{\mu} \in A$, then $a_{\lambda}=h_{\lambda \mu}(f) a_{\mu} \in A$ and there is in $B / I_{\lambda}+I_{\mu}$ an $x$ such that $f(x)=\hat{x}(f) \neq 0$. Thus, if $p_{\lambda}\left(x_{\lambda}\right)=p_{\mu}\left(x_{\mu}\right)=x$, then $x_{\lambda} / \hat{x}(f) \otimes a_{\lambda}$ $\in B_{\lambda} \otimes A, \quad x_{\mu} / \hat{x}(f) \otimes a_{\mu} \in B_{\mu} \otimes A$. Furthermore $\left(x_{\lambda} / \hat{x}(f)\right) \hat{n}(f) a_{\lambda}=a_{\lambda}$, $\left(\hat{x}_{\mu}(f) / \hat{x}(f)\right) h_{\lambda \mu}(f) a_{\mu}=\left(x_{\mu} / \hat{x}(f)\right) \hat{(}(f) h_{\lambda \mu}(f) a_{\mu}$ and so by direct calculation $a_{\mu}^{\prime}=h_{\mu \lambda}^{*}(f) a_{\lambda}$. The definitions imply $\vartheta$ is also surjective and, since $L$ is compact [3], $\vartheta$ is a homeomorphism. Furthermore, $\operatorname{Hom}(D, \mathbf{C})$ is a Hausdorff space [8]. Hence

$$
L=\bigcup_{\lambda} F_{\lambda} \times \operatorname{Hom}(A, \mathbf{C}) / \sim_{\mathscr{B}} \cong \operatorname{Hom}(D, \mathbf{C}) .
$$

Setting $\dot{U}_{\lambda} U_{\lambda} \times \operatorname{Hom}(A, \mathbf{C}) / \sim$ to be the disjoint union (of the $U_{\lambda} \times \operatorname{Hom}(A, \mathbf{C})$ ) reduced by the equivalence relation $\sim$ derived from $\sim \mathscr{B}(\sim$ is the inverse image of 
$\sim_{\mathscr{B}}$ under the canonical injection $\left.\dot{\cup}_{\lambda} U_{\lambda} \times \operatorname{Hom}(A, \mathbf{C}) \hookrightarrow \dot{U}_{\lambda} F_{\lambda} \times \operatorname{Hom}(A, \mathbf{C})\right)$ yields the desired result.

COROllaRY. Let $D^{\prime}$ be the subalgebra consisting of all vectors $\tau=\left\{\tau_{\lambda}\right\}$ in $S$, $\tau_{\lambda}=\sum_{i} x_{\lambda i} \otimes a_{\lambda i}$ in $B_{\lambda} \otimes_{\pi} A$, and such that if $U_{\lambda} \cap U_{\mu} \neq \varnothing$ for some $f$ in $U_{\lambda} \cap U_{\mu}$, $\hat{\tau}_{\lambda}(f)=\sum_{i} \hat{x}_{\lambda i}(f) a_{\lambda i}=\sum_{j} \hat{x}_{\mu j}(f) h_{\lambda \mu}(f) a_{\mu j}$. Then

$$
\operatorname{Hom}\left(D^{\prime}, \mathbf{C}\right) \underset{\text { homeo. }}{\cong} \bigcup_{\lambda} U_{\lambda} \times \operatorname{Hom}(A, \mathbf{C}) / \sim \text {, }
$$

- being the equivalence relation defined above.

REMARK. The algebras $D^{\prime}$ and $D$ differ only in the specifications $f \in U_{\lambda} \cap U_{\mu}$, and $f \in F_{\lambda} \cap F_{\mu}$, respectively.

Proof. If $i$ is the (continuous) injection $\dot{U}_{\lambda} U_{\lambda} \times \operatorname{Hom}(A, \mathbf{C}) / \sim \hookrightarrow \dot{U}_{\lambda} F_{\lambda} \times$ $\operatorname{Hom}(A, \mathbf{C}) / \sim \mathscr{B}$ and if $l=\vartheta \circ i(\vartheta: L \rightarrow \operatorname{Hom}(D, \mathbf{C})$ as defined earlier $)$, then $l$ maps $\dot{U}_{\lambda} U_{\lambda} \times \operatorname{Hom}(A, \mathbf{C})$ into $\operatorname{Hom}(D, \mathbf{C})$. Clearly there is an epimorphism $\xi: D \rightarrow D^{\prime}$ and hence $\operatorname{Hom}\left(D^{\prime}, \mathbf{C}\right)$ may be injected into $\operatorname{Hom}(D, \mathbf{C})$. It follows at once that $\operatorname{Image}(l)=\operatorname{Hom}\left(D^{\prime}, \mathbf{C}\right)$, as required.

3. Elaborations. It is of interest to explore the relation between the notions of a coordinate fibre tensor product bundle and a coordinate bundle in the usual sense. To this end let $\mathscr{E}$ be an arbitrary bundle with base space $\operatorname{Hom}(B, \mathrm{C})$, fibre $\operatorname{Hom}(A, \mathbf{C})$, and group $\mathscr{A}^{*}$ defined as in $\S 2$. Assume that $\mathscr{E}$ is specified by a coordinate bundle such that:

(i) $A$ is a locally convex algebra with identity and compact spectrum; $\operatorname{Aut}(A)$ is an equicontinuous subset of $\mathscr{L}_{s}(A)$.

(ii) $B$ is a locally convex algebra with identity and for some finite open cover $\left\{V_{i}\right\}$ of $\operatorname{Hom}(B, \mathbf{C})$ each homeomorphism $\bar{\varphi}_{V_{i}}: V_{i} \times \operatorname{Hom}(A, \mathbf{C}) \rightarrow p^{-1}\left(V_{i}\right)$ has a unique extension to a fibre-preserving homeomorphism $\varphi_{F_{i}}$ for some compact hull $F_{i} \supset \bar{V}_{i}$.

(iii) Each transition function $\bar{g}_{V_{i} V_{j}}: V_{i} \cap V_{j} \rightarrow \mathscr{A}^{*}$ has a unique continuous extension to a map $g_{F_{i} F_{j}}: F_{i} \cap F_{j} \rightarrow \mathscr{A}^{*}$ so that $\varphi_{F_{i}}(\beta, \alpha)=\varphi_{F_{j}}\left(\beta, g_{F_{i} F_{j}}(\beta)(\alpha)\right)$.

The situation above obtains, e.g., if $A$ and $B$ are the algebras of $C$-valued $\mathscr{C}^{\infty}$-maps on compact differentiable manifolds [9].

LEMMA 3. Each coordinate fibre bundle $\mathscr{E}$ satisfying (i), (ii) and (iii) defines a coordinate fibre tensor product bundle.

Proof. Since $F_{i}=\operatorname{hull}\left(\operatorname{kernel}\left(F_{i}\right)\right)$ [9], if $I_{i}=\operatorname{kernel}\left(F_{i}\right)$ and $B_{i}=B / I_{i}$, then $\operatorname{Hom}\left(B_{i}, \mathbf{C}\right)$ and $F_{i}$ are homeomorphic. Since $\bar{g}_{V_{i} V_{j}}(f) \in \mathscr{A}^{*}$ it follows that there is a $\operatorname{map} \bar{h}_{V_{j} V_{i}}: V_{i} \cap V_{j} \rightarrow \operatorname{Aut}(A)$ so that $\bar{g}_{V_{i} V_{j}}(f)$ is the dual of $\bar{h}_{V_{j} V_{i}}(f)$ for $f$ in $V_{i} \cap V_{j}$. If $\operatorname{Aut}(A)$ is equipped with the compact open (" $c$ ") topology, then $\bar{h}_{V_{i} V_{j}}: V_{i} \cap V_{j} \rightarrow$ Aut ${ }_{c}(A)$ is continuous as is $\bar{h}_{V_{j} V_{i}}: V_{i} \cap V_{j} \rightarrow$ Aut $_{s}(A)$. There are, corresponding to the maps $g_{F_{i} F_{j}}$, maps $h_{F_{j} F_{i}}$ and these serve as the transition maps that can be used to define the algebra $S$ and the subalgebra $D$ as in $\S 1$. (The ideals $I_{\lambda}$ are now $\operatorname{kernel}\left(F_{i}\right)$.)

On the other hand, the maps $h_{F_{j} F_{i}}$ (and their restrictions $\bar{h}_{V_{1} V_{i}}$ ) can be used to define a "standard" locally convex algebra bundle $\tilde{\mathscr{E}}$ with base space $\operatorname{Hom}(B, C)$, fibre $A$, and group $\operatorname{Aut}(A)$. 
If $\Gamma(\tilde{\mathscr{E}})$ is the locally convex algebra of sections of $\tilde{\mathscr{E}}$, then there can be established an isomorphism

$$
k: D \ni\left\{t_{V_{i}}: V_{i} \in\left\{V_{i}\right\}\right\} \mapsto \gamma(f)=\bar{\varphi}_{V_{i}}\left(f, \delta_{V_{i} f}\left(t_{V_{i}}\right)\right) \in \Gamma(\mathscr{E}),
$$

where $f \in V_{i}$ and $\delta_{V_{i} f}: B_{i} \otimes_{\pi} A \rightarrow A: \sum_{m} x_{i}^{m} \otimes a^{m} \mapsto \sum_{m} f\left(x_{i}^{m}\right) a^{m}$ is an "evaluation" map. The proof is outlined as follows:

(a) $k$ is well defined and is independent of the choice of neighborhood containing $f$.

(b) $k$ is 1-1 since $\bar{\varphi}_{V_{i}}$ is a homeomorphism and $\delta_{V_{f}}$, as an evaluation map, is 1-1.

(c) $k$ is surjective: If $\gamma \in \Gamma(\tilde{\mathscr{E}}), t_{V_{i} f}(\gamma(f)) \in A$ and thus $1_{i} \otimes t_{V_{i} f}(\gamma(f)) \in B_{i} \otimes A$ $\left(1_{i}\right.$ is the identity of $\left.B_{i}=B / \operatorname{kernel}\left(F_{i}\right)\right)$. Hence the elements $\left\{1_{i} \otimes t_{v, f}(\gamma(f))\right\} \in D$.

(d) $k$ is bicontinuous if $D$ is given the locally convex topology induced on it by $\sum_{i} B_{i} \otimes_{\pi} A$ and if $\Gamma(\tilde{\mathscr{E}})$ is given the topology described in [7]. Since [7] has not appeared at this writing, a brief description of the topology for $\Gamma(\tilde{\mathscr{E}})$ follows:

For $V_{i}$ open in $\operatorname{Hom}(B, \mathbf{C})$ give the algebra $\Gamma\left(V_{i}, \tilde{\mathscr{E}}\right)$ of sections over $V_{i}$ seminorms

$$
N_{K, p}(\gamma)=\sup _{x \in K} p\left(t_{V_{i}}(\gamma(x))\right) \text {, }
$$

$K$ compact, $K \subset V_{i}, p$ a seminorm from the set of seminorms that topologize $A, t_{V_{1}}$ the usual isomorphism, $p^{-1}(x)=A[5]$.

If $i_{V_{i}}: \Gamma(\tilde{\mathscr{E}}) \rightarrow \Gamma\left(V_{i}, \mathscr{E}\right)$ is the canonical continuous map $\left(\operatorname{Hom}(B, \mathbf{C})=\bigcup_{i} V_{i}\right)$, then with respect to the initial topology defined via $\left\{i_{V_{1}}\right\}, \Gamma(\tilde{\mathscr{E}})$ is a locally convex algebra of the type of $A$.

4. Equivalence classes. In analogy with the notion of equivalence classes of coordinate bundles [10] there can be defined an equivalence of coordinate fibre tensor product bundles. The corresponding equivalence classes define fibre tensor product bundles. The summarizing statement for these concepts and constructions is

THEOREM. If $A, B$ are locally convex algebras, each fibre bundle having base $\operatorname{Hom}(B, \mathbf{C})$, fibre $\operatorname{Hom}(A, \mathbf{C})$ and group $\mathscr{A}^{*}$ may be regarded as $\operatorname{Hom}(D, \mathbf{C}), D$ being a fibre tensor product bundle.

\section{REFERENCES}

1. N. Bourbaki, Theory of sets, Addison-Wesley, Reading, Mass., 1968.

2. T__ Topologie générale, Chapitre X. Hermann, Paris, 1961.

3. J. Dugundji, Topologr, Allyn \& Bacon, Boston, Mass., 1966.

4. B. R. Gelbaum, Tensor products over Banach algebras, Trans. Amer. Math. Soc. 118 (1965), $131-149$

5. Banach algebra bundles, Pacific J. Math. 28 (1969), 337-349.

6. A. Kryiazis, On the spectra of topological A-tensor product A-algebras, Yokohama Math. J. 31 (1983), $47-65$.

7. Locally convex algebra bundles (to appear).

8. A. Mallios, On the spectrum of a topological tensor product of locally convex algebras, Math. Ann. 154 (1964), 171-180.

9. General theory of topological algebras: selected topics (to appear).

10. N. Steenrod, The topology of fibre bundles, Princeton Univ. Press, Princeton, N. J., 1960.

Department of Mathematics, State University of New York, Buffalo, New York 14214

Mathematical Institute, University of Athens, Athens (143), Greece 